\title{
Impact de la stratégie d'apprentissage par projets sur l'acquisition des compétences en leadership parmi les élèves réfugiés palestiniens à Gaza
}

\author{
Safa Migdad ${ }^{1}$, Amjad Joma ${ }^{2}$ et Olivier Arvisais ${ }^{3}$ \\ ${ }^{1}$ United Nations Relief and Works Agency for Palestine Refugees in the Near East, Gaza \\ ${ }^{2}$ A'Sharqiyah University Sultanate of Oman \\ ${ }^{3}$ Université du Québec à Montréal, Québec, Canada
}

\section{Pour citer cet article :}

Migdad, S., Joma, A., et Arvisais, O. (2021). Impact de la stratégie d'apprentissage par projets sur l'acquisition des compétences en leadership parmi les élèves réfugiés palestiniens à Gaza. Didactique, 2(1), 4-39.

Résumé : La présente étude vise à examiner l'efficacité de la stratégie d'apprentissage par projets pour le développement des compétences en leadership des élèves de troisième année primaire. Les questions de recherche ont été traitées en utilisant l'approche expérimentale. Les chercheurs ont sélectionné 76 élèves de troisième année de l'école primaire mixte Al Zaitun de Gaza, et ont échantillonné au hasard deux classes de troisième année. Les participants ont été divisés en deux groupes équivalents, chacun composé de 38 élèves. La stratégie d'apprentissage par projets était utilisée pour enseigner l'anglais au groupe expérimental, tandis que la méthode traditionnelle était utilisée pour le groupe contrôle. L'outil d'étude consistait en une grille d'observation sur laquelle les compétences de leadership de l'élève étaient notées avant et après l'application. Les données ont été analysées à l'aide du logiciel Statistical Package for Social Sciences. Le test $t$ et la taille de l'effet ont été utilisés sur les échantillons pour l'analyse. Les résultats ont révélé des 
notes moyennes plus élevées en matière de compétences en leadership pour le groupe expérimental sur la grille d'observation post-expérimentation. Les chercheurs ont attribué ces résultats à la stratégie d'apprentissage par projets et les résultats de cette recherche tendent à soutenir la recommandation de son utilisation dans l'enseignement de l'anglais pour développer les compétences en leadership. Des recherches supplémentaires sont nécessaires pour étudier l'effet d'autres stratégies d'apprentissage par projets sur d'autres niveaux et habiletés fondamentales.

Mots-clés : apprentissage par projets, compétences en leadership, école primaire, Bande de Gaza, Palestine 


\section{Introduction}

De nos jours, l'apprentissage de la langue anglaise est devenu un besoin indéniable, car l'anglais figure parmi les langues les plus indispensables et les plus prédominantes au monde. De plus, l'anglais est la langue utilisée au quotidien dans de nombreux secteurs tels que les affaires, l'économie, la technologie et l'éducation post-secondaire. « Au cours des dernières décennies, on a observé un besoin accru d'utiliser l'anglais pour exprimer les connaissances dans des domaines professionnels spécifiques »(Ardeo, 2003, p. 110).

Cameron (2001, p. 6) fait remarquer qu'il existe certaines différences entre enseigner une langue étrangère aux enfants et l'enseigner aux adultes. Les enfants sont généralement plus enthousiastes et dynamiques, mais leur intérêt diminue plus rapidement et ils sont moins aptes à demeurer motivés pour accomplir une tâche qu'ils trouvent difficile. Les enfants ont plus de difficulté à maîtriser la langue de façon aussi précise et courante que les apprenants plus âgés, et ils ont tendance à la parler avec un accent moins prononcé. De plus, il leur est également difficile de demeurer concentrés pendant plus de 10 à 20 minutes; ils commencent ensuite à s'ennuyer et à éprouver de la fatigue. Au vu de ces éléments, la pertinence de l'apprentissage actif devient évidente.

De nombreuses stratégies ont été utilisées pour faciliter le processus d'apprentissage et l'enseignement de l'anglais. Afin de relever tous les défis auxquels le processus éducatif fait face concernant l'anglais comme langue seconde, l'enseignant devrait mettre en œuvre des méthodes d'enseignement appropriées qui encouragent les élèves à utiliser la langue étudiée, tout en suscitant leurs intérêts et en améliorant leurs différentes compétences pratiques en leadership, comme la prise de décisions et la pensée critique (National Academy Foundation, 2011).

L'apprentissage par projets (APP) est l'une des nouvelles stratégies d'apprentissage les plus prometteuses, qui répond aux besoins des enfants en matière d'apprentissage actif. Hallermann et coll. (2011, p. 5) définissent l'apprentissage par projets comme une méthode d'enseignement systématique qui encourage les élèves à acquérir les connaissances importantes et les compétences essentielles au 21e siècle, dans le cadre d'un processus d'enquête élargi, influencé par l'élève, structuré autour de questions complexes et authentiques et de produits et tâches d'apprentissage soigneusement conçus. L'apprentissage par projets offre une vaste gamme d'avantages tant aux élèves qu'aux enseignants. De plus en plus de recherches universitaires appuient l'utilisation de l'apprentissage par projets à l'école pour motiver les élèves, diminuer l'absentéisme, stimuler les compétences en apprentissage coopératif et améliorer la performance scolaire (Kokotsaki et al., 2016; Megayanti et al., 2020). De plus, l'apprentissage par projets a montré qu'il bénéficiait à toute une variété d'élèves concernant le développement des 
compétences collaboratives. Par exemple, grâce à l'APP, des élèves du primaire étaient capables de comprendre plusieurs points de vue et d'acquérir des compétences en résolution de conflits (ChanLin, 2008). D'après les enseignants du primaire, l'APP a plusieurs effets positifs sur les connaissances des élèves sur le sujet. Comparativement aux classes traditionnelles, les classes d'APP obtenaient de meilleurs résultats aux évaluations des connaissances du sujet. En outre, Hallermann et coll. (2011, p. 5) stipulent qu'avec l'apprentissage par projets, les élèves doivent prendre part au programme scolaire par le biais d'une question subtile à étudier, d'un problème concret à résoudre ou d'un défi créatif à relever. Avant de pouvoir accomplir ces tâches, les élèves doivent réfléchir au sujet en posant des questions et en élaborant leurs propres réponses. Pour montrer ce qu'ils ont appris, les élèves doivent ensuite créer des produits de grande qualité et présenter leur travail aux autres élèves. Les élèves travaillent souvent sur des sujets en petits groupes, guidés par l'enseignant. Les élèves doivent effectuer leurs propres recherches pour trouver les réponses à des questions qui sont généralement plus difficiles. Pour trouver les réponses, les apprenants doivent réfléchir profondément, effectuer les recherches, recueillir les données nécessaires, établir des prévisions, analyser les données, combiner et interpréter les résultats, évaluer les observations, et finalement présenter les solutions au problème (Baghri et coll, 2013, p. 18).

De telles activités, qu'elles se déroulent en classe ou à l'extérieur de la classe, nécessitent des leaders d'équipe. Les enseignants devraient, par conséquent, encourager les élèves à mettre leurs compétences en leadership en pratique et à comprendre l'importance de les améliorer. Les compétences en leadership sont extrêmement pertinentes pour les élèves à différents niveaux, surtout pour les jeunes apprenants, car ce seront eux qui occuperont les futurs postes de dirigeants. Les élèves réfugiés palestiniens, tout particulièrement, bénéficieraient grandement de ces compétences en leadership étant donné les circonstances difficiles et les situations d'urgence auxquelles ils doivent faire face. En classe, les enseignants peuvent aider leurs élèves à mettre en pratique plusieurs compétences en leadership en les faisant participer à différents exercices tels que les activités de planification, la désignation des leaders de groupe, la possibilité de jouer le rôle d'enseignant et de faire des présentations devant la classe. De plus, les enseignants doivent activement s'efforcer d'améliorer ces compétences en leadership très tôt dans le processus de perfectionnement pédagogique des élèves. Moody (2000) fait remarquer que les apprenants qui manifestent des comportements de leadership sont plus aptes à faire face aux difficultés et aux défis. Par conséquent, en procurant aux enseignants des ressources en développement du leadership, notamment des livres, des vidéos, des logiciels et des ateliers, on pourrait les aider à instaurer des environnements éducatifs qui permettraient aux élèves de devenir des leaders efficaces. 
De nombreuses études se sont penchées sur l'efficacité de l'apprentissage par projets, tant pour l'étude de l'anglais que pour d'autres types d'apprentissage (Kokotsaki et al., 2016). Ces études appuient l'efficacité de cette stratégie en ce qui a trait à la réussite scolaire et au développement de différentes habiletés fondamentales (Megayanti et al., 2020). Par exemple, Bagheri et coll. (2013) ont rapporté les avantages que présentait cette stratégie pour les aptitudes à l'apprentissage autonome des élèves, alors que Baş (2011) et d'autres ont rapporté les avantages de l'apprentissage par projets pour la réussite scolaire des élèves et leurs comportements à l'endroit de l'anglais (Chen \& Yang, 2019).

La présente étude vise à évaluer les effets de la stratégie d'apprentissage par projets sur le développement des compétences en leadership parmi les élèves de troisième année des écoles primaires de l'UNRWA à Gaza.

\section{Énoncé du problème}

Le problème visé par l'étude est énoncé dans la question générale suivante : Quel est l'impact de la stratégie d'apprentissage par projets sur l'acquisition des compétences en leadership parmi les élèves réfugiés palestiniens à Gaza?

\section{Questions de recherche}

1. Quelles sont les compétences en leadership qui doivent être développées au moyen de la stratégie APP?

2. Quelles sont les différences statistiquement significatives $(\alpha \leq 0,05)$ dans le niveau des compétences en leadership entre les élèves du groupe expérimental et ceux du groupe contrôle aux stades finaux de l'expérimentation?

3. Quelles sont les différences statistiquement significatives $(\alpha \leq 0,05)$ dans le niveau des compétences en leadership des élèves du groupe expérimental entre les stades initial et final de l'expérimentation?

4. Quelles sont les différences statistiquement significatives $(\alpha \leq 0,05)$ dans le niveau des compétences en leadership entre les élèves de sexe masculin et les élèves de sexe féminin du groupe expérimental?

\section{Objectif de l'étude}

L'étude vise à mesurer l'impact de l'utilisation de la stratégie APP sur l'acquisition des compétences en leadership parmi les élèves réfugiés palestiniens à Gaza. 


\section{Limites de l'étude}

L'étude présente certaines limites suivantes. D'abord, elle se concentre seulement sur les élèves de troisième année des écoles de l'UNRWA. Ensuite, elle s'est déroulée durant le premier semestre de l'année scolaire 2015-2016. De plus, elle se limite aux écoles primaires de la partie est de Gaza. Finalement, l'expérimentation n'utilise que trois unités de l'ouvrage English for Palestine.

\section{Définition opérationnelle des termes}

Avant d'aborder le dispositif méthodologique, certaines définitions doivent être établies.

Dans un premier temps, la stratégie d'apprentissage par projets (APP) est un modèle d'activités effectuées en classe qui se distingue du modèle habituel de leçons courtes, isolées et centrées sur l'enseignant se pratiquant habituellement dans les salles de classe. Nous utilisons ici la définition proposée par Proulx (2004, p. 31) :

Un processus systématique d'acquisition et de transfert de connaissances au cours duquel l'apprenant anticipe, planifie et réalise, dans un temps déterminé, seul ou avec des pairs et sous supervision d'un enseignant, une activité observable qui résulte, dans un contexte pédagogique, en un produit fini évaluable.

En complément de cette première définition, Chiung-Sui Chang et coll. (2011) définissent également l'APP comme une stratégie d'enseignement qui forme les apprenants sur la façon d'utiliser les connaissances, les compétences et les comportements acquis pour résoudre les problèmes et s'adapter aux circonstances imprévues qui se présentent dans la vie réelle.

Dans le présent document, la stratégie APP est conceptualisée sous forme d'un ensemble d'activités se déroulant à l'intérieur ou à l'extérieur de la classe. Ces activités sont dirigées par les apprenants qui travaillent en groupes pour résoudre certains problèmes, afin d'apprendre plus efficacement le nouveau vocabulaire et améliorer leurs compétences en leadership.

Dans un deuxième temps, les compétences en leadership pour Potter (1997, p. 6), consistent à susciter des sentiments positifs de la part des personnes dirigées et à mettre l'accent sur l'inspiration plutôt que sur le contrôle. Gill (2012, p. 101) définit également le leadership comme la capacité d'accomplir des choses utiles et souhaitables qui bénéficient aux personnes dirigées.

Dans le présent document, les compétences en leadership sont conceptualisées sous la forme d'un ensemble de compétences que les enfants devraient posséder pour pouvoir 
influencer les autres afin d'atteindre des buts communs. Ces compétences comprennent la planification de projet, l'aptitude à communiquer, la résolution de problèmes, la prise de décisions, la confiance en soi, les habiletés en matière de présentation, la gestion du temps, le travail d'équipe, l'efficacité personnelle, l'esprit d'initiative et la prise de responsabilités. La stratégie APP améliore ces compétences qui sont mesurées par les notes des élèves indiquées sur la grille d'observation du leadership.

\section{Méthodologie}

\section{Conception de la recherche}

L'étude utilisait une approche expérimentale qui nécessitait deux groupes d'élèves, un groupe expérimental et un groupe contrôle. La stratégie d'apprentissage par projets était appliquée pour enseigner au groupe expérimental, tandis que la méthode traditionnelle était utilisée avec le groupe contrôle. L'expérimentation s'est déroulée sur six semaines. Les deux groupes avaient comme enseignante la chercheuse principale.

\section{Population participant à l'étude}

La population participant à l'étude est composée de tous les élèves de troisième année des écoles de l'UNRWA de Gaza.

\section{Échantillon de l'étude}

L'échantillon de l'étude est composé de 76 élèves répartis en deux groupes : le groupe expérimental et le groupe contrôle, qui comprenaient 38 élèves chacun. L'échantillon de l'étude est constitué des participants volontaires choisi dans l'école «C» d'Al Zaitun Elem Co-ed, située dans l'est de Gaza, dans laquelle la chercheure principale était professeure d'anglais. L'échantillon a été choisi de façon aléatoire parmi les classes de troisième année. Le tableau 2.1 montre la répartition de l'échantillon.

Tableau 2.1 : Répartition de l'échantillon

\begin{tabular}{cccc}
\hline Groupe & Expérimental & Contrôle & Total \\
\hline \hline Nombre d'élèves & 38 & 38 & 76 \\
\hline
\end{tabular}

\section{Instruments de l'étude - Grille d'observation}

Une grille d'observation (annexe 1) a été préparée par les chercheurs pour mesurer l'impact de la stratégie d'apprentissage par projets sur les compétences en leadership des élèves de 
troisième année. Cette grille d'observation a été utilisée avant et après l'expérimentation avec les deux groupes, le groupe expérimental et le groupe contrôle. Elle comprend 11 domaines totalisant 37 énoncés. Une échelle à cinq niveaux de Likert a été utilisée pour quantifier les observations. Les réponses variaient de pas du tout d'accord, pas d'accord, ni d'accord ni pas d'accord, d'accord à tout à fait d'accord. Voici la liste des compétences présentes sur la grille d'observation :

1. Planification : capacité de l'élève à se préparer à ce qu'il aura à faire dans le cadre d'un projet, notamment définir les objectifs, préparer le matériel et établir les étapes du projet.

2. Aptitude à communiquer : capacité de l'élève à utiliser ses aptitudes verbales, non verbales et en communication écrite de façon efficace pour transmettre des idées et faire part de ses opinions et de ses sentiments.

3. Résolution de problèmes : capacité de l'élève à proposer et à créer plusieurs solutions pour résoudre des situations problématiques.

4. Prise de décisions : capacité de l'élève à percevoir les choses à partir de différents points de vue et de prendre des décisions éthiques ou de tenir compte du point de vue des autres.

5. Confiance en soi : capacité de l'élève à découvrir ses intérêts personnels et ses héros pour trouver l'inspiration et la motivation.

6. Habiletés en matière de présentation : capacité de l'élève à présenter et à montrer son projet (titre, buts, importance, procédures et résultats) d'une façon systémique et logique en utilisant des graphiques, des photos, des diagrammes et des cartes conceptuelles.

7. Gestion du temps : capacité de l'élève à gérer et à organiser son temps et son travail dans le cadre des activités et des tâches du projet.

8. Travail d'équipe : capacité de l'élève à faire en sorte que les choses soient faites sans se montrer autoritaire ou s'aliéner les autres. Les bons leaders apprennent des autres et acceptent d'être dirigés.

9. Efficacité personnelle : capacité de l'élève à influencer les autres en les motivant pour qu'ils fassent quelque chose ou en les persuadant de penser autrement.

10. Esprit d'initiative : capacité de l'élève à faire les choses de façon autonome, sans demander de l'aide, et à agir plutôt qu'attendre.

11. Prise de responsabilités : capacité de l'élève à agir et à se comporter de façon appropriée quand on lui offre l'occasion d'assumer des responsabilités et de faire 
l'expérience de nombreux et différents rôles au sein de clubs, durant des voyages scolaires, au cours de présentations ou de débats, et chez lui.

La version finale de l'échelle consistait en 37 énoncés répartis dans 11 domaines, tel qu'illustré dans le tableau 2.2 ci-dessous.

Tableau 2.2 : Domaines de la grille d'observation

\begin{tabular}{lc}
\hline \multicolumn{1}{c}{ Domaines } & Nombre d'énoncés \\
\hline \hline Compétences en planification & 4 \\
Aptitude à communiquer & 4 \\
Compétences en résolution de problèmes & 3 \\
Prise de décisions & 3 \\
Confiance en soi & 4 \\
Habiletés en matière de présentation & 3 \\
Compétences en gestion du temps & 3 \\
Travail d'équipe/en groupe & 3 \\
Efficacité personnelle & 4 \\
Esprit d'initiative & 3 \\
Prise de responsabilités & 3 \\
\hline Total & 27 \\
\hline
\end{tabular}

Étude pilote. La grille d'observation a tout d'abord été utilisée sur un échantillon pilote aléatoire composé de cinq élèves de troisième année de l'école « $\mathrm{C}$ » d'Al Zaitun Elem Coed située dans l'Est de Gaza, en vue de s'assurer que les énoncés et les instructions qu'elle comprenait étaient clairs. Cela a également servi à évaluer la fidélité et la fiabilité de la grille d'observation.

Fidélité de la grille d'observation. Les chercheurs ont utilisé deux méthodes de fidélité, celle des examinateurs et celle de la cohérence interne.

Fidélité des examinateurs. La grille d'observation était présentée à un jury composé de superviseurs expérimentés et de spécialistes de la langue anglaise, de la méthodologie et de la psychologie, de professeurs de l'université de Gaza et de représentants du ministère 
de l'Éducation. Les énoncés de la grille d'observation étaient modifiés en fonction de leurs recommandations.

La cohérence interne. L'analyse de la cohérence interne montre la corrélation entre la note attribuée à chaque énoncé et la note totale des domaines. Elle montre aussi la corrélation entre la moyenne de chaque domaine et la moyenne totale. Cette fidélité a été calculée à l'aide de la formule de Pearson. Le tableau 2.3 montre la valeur de cohérence interne de la grille d'observation.

D'après le tableau 2.3, le coefficient de corrélation de chaque énoncé, dans chaque domaine, est significatif au niveau 0,01 . Le tableau 2.4 montre le coefficient de corrélation de chaque domaine avec l'ensemble de la grille d'observation. En nous basant sur les tableaux suivants, nous avons conclu que la grille d'observation était fortement cohérente et fiable en tant qu'outil d'étude. 
Tableau 2.3 : Coefficient de corrélation Pearson pour chaque domaine avec le degré total de la grille d'observation

\begin{tabular}{|c|c|c|c|c|c|}
\hline Domaines & Énoncés & $\begin{array}{l}\text { Corrélation } \\
\text { Pearson }\end{array}$ & Domaines & Énoncés & $\begin{array}{l}\text { Corrélation } \\
\text { Pearson }\end{array}$ \\
\hline \multirow{4}{*}{ Planification } & 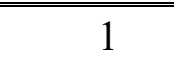 & $0,969 * *$ & \multirow{3}{*}{$\begin{array}{l}\text { Habiletés en } \\
\text { matière de } \\
\text { présentation }\end{array}$} & 19 & $0,966^{* *}$ \\
\hline & 2 & $0,980^{* *}$ & & 20 & $0,980^{* *}$ \\
\hline & 3 & $0,976^{* *}$ & & 21 & $0,977^{* *}$ \\
\hline & 4 & $0,966^{* *}$ & \multirow{3}{*}{\begin{tabular}{|l} 
Gestion du \\
temps
\end{tabular}} & 22 & $0,959^{* *}$ \\
\hline \multirow{4}{*}{$\begin{array}{l}\text { Aptitude à } \\
\text { communiquer }\end{array}$} & 5 & $0,935 * *$ & & 23 & $0,972^{* *}$ \\
\hline & 6 & $0,954^{* *}$ & & 24 & $0,969^{* *}$ \\
\hline & 7 & $0,948^{* *}$ & \multirow{3}{*}{$\begin{array}{l}\text { Travail } \\
\text { d'équipe }\end{array}$} & 25 & $0,956^{* *}$ \\
\hline & 8 & $0,914 * *$ & & 26 & $0,984 * *$ \\
\hline \multirow{3}{*}{$\begin{array}{l}\text { Résolution de } \\
\text { problèmes }\end{array}$} & 9 & $0,956^{* *}$ & & 27 & $0,984^{* *}$ \\
\hline & 10 & $0,979 * *$ & \multirow{5}{*}{$\begin{array}{l}\text { Efficacité } \\
\text { personnelle }\end{array}$} & 28 & $0,960^{* *}$ \\
\hline & 11 & $0,965^{* *}$ & & 29 & $0,976^{* *}$ \\
\hline \multirow{3}{*}{$\begin{array}{ll}\text { Prise de } \\
\text { décisions }\end{array}$} & 12 & $0,970 * *$ & & 30 & $0,967^{* *}$ \\
\hline & 13 & $0,989 * *$ & & 31 & $0,973^{* *}$ \\
\hline & 14 & $0,946^{* *}$ & & 32 & $0,961 * *$ \\
\hline \multirow{5}{*}{$\begin{array}{l}\text { Confiance en } \\
\text { soi }\end{array}$} & 15 & $0,958 * *$ & \multirow{2}{*}{$\begin{array}{l}\text { Esprit } \\
\text { d'initiative }\end{array}$} & 33 & $0,974 * *$ \\
\hline & 16 & $0,958 * *$ & & 34 & $0,973^{* *}$ \\
\hline & 17 & $0,924 * *$ & \multirow{3}{*}{$\begin{array}{l}\text { Prise de } \\
\text { responsabili } \\
\text { tés }\end{array}$} & 35 & $0,977^{* *}$ \\
\hline & 18 & $0,936^{* *}$ & & 36 & $0,990^{* *}$ \\
\hline & & & & 37 & $0,988^{* *}$ \\
\hline
\end{tabular}

Valeur de la table $r$ au dl (degré de liberté) 38 et au niveau de signification $0,05=0,304$ Valeur de la table $r$ au dl (degré de liberté) 38 et au niveau de signification 0,01 =0,393

Tableau 2.4 : Coefficient de corrélation de chaque domaine de la grille d'observation avec le total

\begin{tabular}{lcc}
\hline \multicolumn{1}{c}{ Domaine } & Corrélation Pearson & Niveau de signification \\
\hline \hline Planification & 0,933 & sig. à 0,01 \\
Aptitude à communiquer & 0,965 & sig. à 0,01 \\
$\begin{array}{l}\text { Compétences en résolution de } \\
\text { problèmes }\end{array}$ & 0,935 & sig. à 0,01 \\
Prise de décisions & 0,918 & sig. à 0,01
\end{tabular}




$\begin{array}{llr}\text { Confiance en soi } & 0,921 & \text { sig. à } 0,01 \\ \text { Habiletés en matière de présentation } & 0,950 & \text { sig. à } 0,01 \\ \text { Compétences en gestion du temps } & 0,933 & \text { sig. à } 0,01 \\ \text { Travail d'équipe/en groupe } & 0,939 & \text { sig. à } 0,01 \\ \text { Efficacité personnelle } & 0,945 & \text { sig. à } 0,01 \\ \text { Esprit d'initiative } & 0,948 & \text { sig. à } 0,01 \\ \text { Prise de responsabilités } & 0,949 & \text { sig. à } 0,01\end{array}$

Valeur de la table $\mathrm{r}$ au dl (degré de liberté) 38 et au niveau de signification $0,05=0,304$

Valeur de la table $\mathrm{r}$ au dl (degré de liberté) 38 et au niveau de signification $0,01=0,393$

Fiabilité de la grille d'observation. L'outil est considéré comme fiable s'il donne les mêmes résultats lorsqu'il est réutilisé dans les mêmes conditions. La fiabilité de la grille d'observation a été mesurée avec le coefficient alpha de Cronbach, en utilisant la technique d'homogénéité et l'accord inter-juges.

Coefficient de l'accord interexaminateurs. Pour mesurer la fiabilité de la grille d'observation, nous avons utilisé la méthode de l'accord inter-juges (la chercheuse principale et une autre enseignante). Chaque examinateur travaillait indépendamment et utilisait la même échelle pour consigner la performance des élèves pendant la période d'observation. La chercheuse principale et l'autre enseignante faisaient leurs observations au même moment, soit à la fin de la période spécifiée de l'observation totale. La chercheuse principale et l'autre enseignante observaient cinq élèves. Le coefficient d'accord était calculé statistiquement à l'aide de l'équation de Cooper; les résultats sont indiqués dans le tableau 2.5 ci-dessous.

$$
\text { Coefficient d'accord }=\quad \frac{\text { Points d'accord }}{\text { Points d'accord + Points de désaccord }}
$$

Tableau 2.5: Pourcentage d'accord inter-juges pour évaluer la fiabilité de la grille d'observation

\begin{tabular}{ccccc}
\hline Groupe & $\begin{array}{c}\text { Performance } \\
\text { totale }\end{array}$ & $\begin{array}{c}\text { Premier } \\
\text { examinateur }\end{array}$ & $\begin{array}{c}\text { Second } \\
\text { examinateur }\end{array}$ & Pourcentage \\
\hline \hline Élève 1 & 185 & 133 & 124 & 93,23 \\
Élève 2 & 185 & 127 & 118 & 92,91 \\
Élève 3 & 185 & 115 & 106 & 92,17
\end{tabular}




$\begin{array}{lrccc}\text { Élève } 4 & 185 & 111 & 99 & 89,19 \\ \text { Élève 5 } & 185 & 121 & 115 & 95,04 \\ \text { Fiabilité totale de la grille } & & & & 92,51\end{array}$

Selon le tableau 2.5, nous avons trouvé que le pourcentage d'accord inter-juges le plus élevé était de 95,04, que le pourcentage d'accord le plus bas était de 89,19, et que la fiabilité totale était de 92,51. Ces pourcentages indiquent un niveau élevé de fiabilité inter-juges.

Techniques d'homogénéité. Selon les tableaux 2.6 et 2.7 , la grille d'observation s'est révélée fiable, avec un coefficient alpha de Cronbach de 0,994 et un coefficient d'homogénéité de 0,969 .

Tableau 2.6 : Coefficients alpha de Cronbach des domaines de la grille d'observation

\begin{tabular}{ccc}
\hline Alpha de Cronbach & Total & Domaine \\
\hline \hline 0,981 & 4 & Planification \\
0,947 & 4 & $\begin{array}{c}\text { Aptitude à communiquer } \\
\text { Compétences en résolution de } \\
\text { problèmes }\end{array}$ \\
0,961 & 3 & Prise de décisions \\
0,967 & 3 & Confiance en soi \\
0,959 & 4 & Habiletés en matière de présentation \\
0,973 & 3 & Compétences en gestion du temps \\
0,964 & 3 & Travail d'équipe/en groupe \\
0,974 & 3 & Efficacité personnelle \\
0,977 & 4 & Esprit d'initiative \\
0,967 & 3 & Prise de responsabilités \\
0,985 & 3 & Total \\
\hline 0,994 & 37 & .
\end{tabular}


Tableau 2.7 : Coefficient de fiabilité par la technique d'homogénéité

\begin{tabular}{cccc}
\hline Portée & Total & Coefficient de corrélation & Fiabilité \\
\hline \hline Planification & 4 & 0,937 & 0,967 \\
Aptitude à communiquer & 4 & 0,900 & 0,947 \\
$\begin{array}{c}\text { Compétences en résolution de } \\
\text { problèmes }\end{array}$ & 3 & 0,829 & 0,965 \\
Prise de décisions & 3 & 0,881 & 0,943 \\
Confiance en soi & 4 & 0,905 & 0,950 \\
Habiletés en matière de & 3 & 0,879 & 0,976 \\
présentation & 3 & 0,877 & 0,966 \\
Compétences en gestion du temps & 3 & 0,881 & 0,984 \\
Travail d'équipe/en groupe & 3 & 0,959 & 0,979 \\
Efficacité personnelle & 4 & 0,851 & 0,973 \\
Esprit d'initiative & 3 & 0,876 & 0,988 \\
Prise de responsabilités & 3 & 0,968 & 0,969 \\
\hline Total & 37 & & \\
\hline
\end{tabular}

Contrôle des compétences en leadership précédentes. Afin de s'assurer que les sujets de l'échantillon étaient équivalents dans leurs compétences en leadership précédentes, le chercheur a rempli la grille d'observation avant l'expérimentation. Les résultats des sujets étaient consignés et statistiquement analysés à l'aide du test $t$. Le tableau 2.8 montre la moyenne et l'écart-type de chaque groupe pour chaque compétence en leadership précédente.

Tableau 2.8 : Résultats du test $\mathrm{t}$-Contrôle de la variable des compétences précédentes en leadership

\begin{tabular}{lccccccc}
\hline \multicolumn{1}{c}{ Domaine } & Groupe & $\mathrm{N}$ & Moyenne & Écart-type & $t$ & $\begin{array}{c}\text { Valeur de } \\
\text { signification }\end{array}$ & $\begin{array}{c}\text { Niveau de } \\
\text { signification }\end{array}$ \\
\hline \hline \multirow{2}{*}{ Planification } & Expérimental & 38 & 11,316 & 3,662 & 0,398 & 0,692 & Aucune sig. \\
& Contrôle & 38 & 11,737 & 5,401 & & & \\
Aptitude à & Expérimental & 38 & 11,553 & 3,151 & 0,123 & 0,902 & Aucune sig. \\
communiquer & Contrôle & 38 & 11,658 & 4,206 & & & \\
Résolution de & Expérimental & 38 & 7,605 & 2,955 & 0,253 & 0,801 & Aucune sig. \\
problèmes & Contrôle & 38 & 7,421 & 3,382 & & & \\
\hline
\end{tabular}




\begin{tabular}{lccccccc}
\hline Prise de & Expérimental & 38 & 7,263 & 2,544 & 0,291 & 0,772 & Aucune sig. \\
décisions & Contrôle & 38 & 7,079 & 2,954 & & & \\
Confiance en & Expérimental & 38 & 11,684 & 3,103 & 0,581 & 0,563 & Aucune sig. \\
soi & Contrôle & 38 & 11,211 & 3,953 & & & \\
Habiletés en & Expérimental & 38 & 8,158 & 2,727 & 0,034 & 0,973 & Aucune sig. \\
matière de & Contrôle & 38 & 8,184 & 3,965 & & & \\
présentation & Expérimental & 38 & 8,526 & 2,128 & 0,081 & 0,936 & Aucune sig. \\
Gestion du & Contrôle & 38 & 8,474 & 3,391 & & & \\
temps & Expérimental & 38 & 9,395 & 2,636 & 0,076 & 0,940 & Aucune sig. \\
$\begin{array}{l}\text { Travail } \\
\text { d'équipe/en }\end{array}$ & Contrôle & 38 & 9,342 & 3,387 & & & \\
groupe & Expérimental & 38 & 10,289 & 2,710 & 0,256 & 0,798 & Aucune sig. \\
Efficacité \\
personnelle & Contrôle & 38 & 10,526 & 5,007 & & & \\
Esprit & Expérimental & 38 & 8,579 & 2,213 & 0,224 & 0,823 & Aucune sig. \\
d'initiative & Contrôle & 38 & 8,421 & 3,732 & & & \\
$\begin{array}{l}\text { Prise de } \\
\text { responsabilités }\end{array}$ & Expérimental & 38 & 8,737 & 2,901 & 0,495 & 0,622 & Aucune sig. \\
& Contrôle & 38 & 8,395 & 3,115 & & & \\
Degré total & Expérimental & 38 & 103,105 & 23,625 & 0,092 & 0,927 & Aucune sig. \\
\hline Contrôle & 38 & 102,447 & 36,994 & & & \\
\hline
\end{tabular}

Valeur de la table $t$ au dl (degré de liberté) 74 et au niveau de signification $0,05=2,00$ Valeur de la table $t$ au dl (degré de liberté) 74 et au niveau de signification $0,01=2,66$

L'analyse des résultats a indiqué qu'il n'y avait aucune différence statistiquement significative entre le groupe expérimental et le groupe contrôle à $\leq 0,05$.

Contrôle par rapport au sexe. Pour effectuer le contrôle du niveau de compétence en leadership en fonction du sexe des élèves dans le groupe expérimental, nous avons utilisé le test $t$ des échantillons indépendants, dont les résultats sont indiqués dans le tableau 2.9 ci-dessous. 
Tableau 2.9 : Résultats des tests t des échantillons indépendants - Différences entre les élèves de sexe masculin et les élèves de sexe féminin du groupe expérimental

\begin{tabular}{|c|c|c|c|c|c|c|c|}
\hline Compétence & Sexe & $\mathrm{N}$ & Moyenne & Écart-type & $t$ & $\begin{array}{l}\text { Valeur de } \\
\text { signification }\end{array}$ & $\begin{array}{l}\text { Niveau de } \\
\text { signification }\end{array}$ \\
\hline \multirow{2}{*}{ Planification } & Masc. & 16 & 11,375 & 3,384 & \multirow{2}{*}{0,084} & \multirow{2}{*}{0,934} & \multirow{2}{*}{ Aucune sig. } \\
\hline & Fém. & 22 & 11,273 & 3,930 & & & \\
\hline \multirow{2}{*}{$\begin{array}{l}\text { Aptitude à } \\
\text { communiquer }\end{array}$} & Masc. & 16 & 11,438 & 2,421 & \multirow{2}{*}{0,190} & \multirow{2}{*}{0,851} & \multirow{2}{*}{ Aucune sig. } \\
\hline & Fém. & 22 & 11,636 & 3,646 & & & \\
\hline \multirow{2}{*}{$\begin{array}{l}\text { Compétences en } \\
\text { résolution de } \\
\text { problèmes }\end{array}$} & Masc. & 16 & 7,875 & 3,324 & \multirow{2}{*}{0,475} & \multirow{2}{*}{0,638} & \multirow{2}{*}{ Aucune sig. } \\
\hline & Fém. & 22 & 7,409 & 2,720 & & & \\
\hline \multirow{2}{*}{ Prise de décisions } & Masc. & 16 & 7,250 & 2,955 & \multirow{2}{*}{0,027} & \multirow{2}{*}{0,979} & \multirow{2}{*}{ Aucune sig. } \\
\hline & Fém. & 22 & 7,273 & 2,272 & & & \\
\hline \multirow{2}{*}{ Confiance en soi } & Masc. & 16 & 11,125 & 2,604 & \multirow{2}{*}{0,946} & \multirow{2}{*}{0,350} & \multirow{2}{*}{ Aucune sig. } \\
\hline & Fém. & 22 & 12,091 & 3,421 & & & \\
\hline \multirow{2}{*}{$\begin{array}{l}\text { Habiletés en } \\
\text { matière de } \\
\text { présentation }\end{array}$} & Masc. & 16 & 8,313 & 2,774 & \multirow[b]{2}{*}{0,294} & \multirow[b]{2}{*}{0,770} & \multirow[b]{2}{*}{ Aucune sig. } \\
\hline & Fém. & 22 & 8,045 & 2,751 & & & \\
\hline \multirow{2}{*}{$\begin{array}{l}\text { Compétences en } \\
\text { gestion du temps }\end{array}$} & Masc. & 16 & 8,938 & 1,769 & \multirow{2}{*}{1,016} & \multirow{2}{*}{0,316} & \multirow{2}{*}{ Aucune sig. } \\
\hline & Fém. & 22 & 8,227 & 2,349 & & & \\
\hline \multirow{2}{*}{$\begin{array}{l}\text { Travail } \\
\text { d'équipe/en } \\
\text { groupe }\end{array}$} & Masc. & 16 & 9,625 & 2,446 & \multirow[b]{2}{*}{0,454} & \multirow[b]{2}{*}{0,652} & \multirow[b]{2}{*}{ Aucune sig. } \\
\hline & Fém. & 22 & 9,227 & 2,810 & & & \\
\hline \multirow{2}{*}{$\begin{array}{l}\text { Efficacité } \\
\text { personnelle }\end{array}$} & Masc. & 16 & 10,500 & 1,789 & 0.404 & 0689 & Aucune sio \\
\hline & Fém. & 22 & 10,136 & 3,256 & 0,404 & & \\
\hline Esnrit d'initiative & Masc. & 16 & 8,625 & 2,094 & 0108 & 0015 & Aucune sio \\
\hline Espiti a intirative & Fém. & 22 & 8,545 & 2,345 & 0,100 & , & Auctile sig. \\
\hline Prise de & Masc. & 16 & 8,875 & 2,705 & 0.247 & 0.806 & Aucune sig \\
\hline responsabilités & Fém. & 22 & 8,636 & 3,094 & & 0,000 & \\
\hline Compétences en & Masc. & 16 & 103,938 & 17,819 & 0183 & 0856 & Awune sio \\
\hline leadership & Fém. & 22 & 102,500 & 27,490 & 0,183 & 0,850 & Alv uint sig. \\
\hline
\end{tabular}

Valeur de la table $t$ au dl (degré de liberté) 36 et au niveau de signification $0,05=2,02$ Valeur de la table $t$ au dl (degré de liberté) 36 et au niveau de signification $0,01=2,70$

L'analyse des résultats indique qu'il n'y avait aucune différence statistiquement significative du niveau de compétence en leadership entre les élèves de sexe masculin et les élèves de sexe féminin dans le groupe expérimental à $\leq 0,05$.

\section{Analyse statistique}


Notre équipe a utilisé plusieurs outils statistiques adaptés à la nature de l'étude. D'abord, la technique de l'alpha de Cronbach a été utilisée pour mesurer la fiabilité de la grille d'observation. Ensuite, le Test $t$ des échantillons indépendants a été employé pour contrôler les variables interférentielles et mesurer les différences statistiques des moyennes entre les deux groupes pour ce qui est des variables de l'étude. De plus, le $t, \eta^{2}$, et le $d$ de Cohen ont été utilisés pour vérifier la taille de l'effet des différences que la variable indépendante, c.-à-d. l'intervention, a eu sur la variable dépendante, c.-à-d le groupe expérimental. Finalement, les données ont été collectées à l'aide du logiciel Statistical Package for Social Sciences.

\section{Résultats}

La présente étude avait pour objet d'examiner l'impact de la stratégie d'apprentissage par projets sur les compétences en leadership des élèves de troisième année. Cette section réexamine les questions de recherche et les hypothèses à la lumière des résultats, après l'analyse Pour analyser les données, nous avons utilisé différentes formules statistiques telles que les fréquences, les moyennes, les écarts types et les tests $t$. De plus, nous avons utilisé la taille de l'effet au moyen des valeurs $\eta^{2}$ et $d$ pour calculer la mesure dans laquelle la variable indépendante - la stratégie d'apprentissage par projets - avait un effet sur la variable dépendante - les compétences en leadership du groupe expérimental.

\section{Réponses aux questions de recherche}

Réponse à la première question de recherche. La première question est formulée comme suit: Quelles sont les compétences en leadership qui doivent être développées au moyen de la stratégie APP? Pour répondre à cette question, nous avons dressé la liste des compétences en leadership les plus courantes selon les études connexes (Jokinen, 2005). Aussi, des spécialistes en éducation et en psychologie ont été consultés. Voici la liste des compétences retenues: planification; aptitude à communiquer; résolution de problèmes; prise de décisions; confiance en soi; habiletés en matière de présentation; gestion du temps; travail d'équipe; efficacité personnelle; esprit d'initiative; prise de responsabilités.

Réponse à la deuxième question de recherche. La deuxième question était la suivante : Quelles sont les différences statistiquement significatives $(\alpha \leq 0,05)$ dans le niveau des compétences en leadership entre les élèves du groupe expérimental et ceux du groupe contrôle aux stades finaux de l'expérimentation? Pour répondre à cette question, le chercheur a testé l'hypothèse nulle selon laquelle il n'y a aucune différence statistiquement significative à $\alpha \leq 0,05$ entre le niveau des compétences en leadership des élèves du groupe 
expérimental et celui de leurs pairs du groupe contrôle sur la grille d'observation postexpérimentation. Pour examiner cette hypothèse, les moyennes et les écarts-types des résultats des deux groupes ont été calculés sur la grille d'observation post-expérimentation. Un test $t$ des échantillons indépendants a été utilisé pour évaluer les différences. Les résultats sont présentés dans le tableau 3.1.

Tableau 3.1 : Résultats des tests $t$ des échantillons indépendants - Différences entre le groupe expérimental et le groupe contrôle sur la grille d'observation post-expérimentation

\begin{tabular}{|c|c|c|c|c|c|c|c|}
\hline Compétence & Groupe & $\mathrm{N}$ & Moyenne & Écart-type & $t$ & $\begin{array}{l}\text { Valeur de } \\
\text { signification }\end{array}$ & $\begin{array}{l}\text { Niveau de } \\
\text { signification }\end{array}$ \\
\hline \multirow{2}{*}{ Planification } & Expérimental & 38 & 16,211 & 3,580 & \multirow{2}{*}{4,339} & \multirow{2}{*}{0,000} & \multirow{2}{*}{ sig. à 0,01} \\
\hline & Contrôle & 38 & 12,026 & 4,745 & & & \\
\hline \multirow{2}{*}{$\begin{array}{l}\text { Aptitude à } \\
\text { communiquer }\end{array}$} & Expérimental & 38 & 16,500 & 3,415 & \multirow{2}{*}{4,388} & \multirow{2}{*}{0,000} & \multirow{2}{*}{ sig. à 0,01} \\
\hline & Contrôle & 38 & 12,211 & 4,966 & & & \\
\hline \multirow{2}{*}{$\begin{array}{l}\text { Résolution de } \\
\text { problèmes }\end{array}$} & Expérimental & 38 & 11,263 & 2,956 & \multirow{2}{*}{4,594} & \multirow{2}{*}{0,000} & \multirow{2}{*}{ sig. à 0,01} \\
\hline & Contrôle & 38 & 7,868 & 3,465 & & & \\
\hline \multirow{2}{*}{$\begin{array}{l}\text { Prise de } \\
\text { décisions }\end{array}$} & Expérimental & 38 & 11,421 & 2,500 & \multirow{2}{*}{3,922} & \multirow{2}{*}{0,000} & \multirow{2}{*}{ sig. à 0,01} \\
\hline & Contrôle & 38 & 8,421 & 3,998 & & & \\
\hline \multirow{2}{*}{$\begin{array}{l}\text { Confiance en } \\
\text { soi }\end{array}$} & Expérimental & 38 & 16,289 & 3,502 & \multirow{2}{*}{3,790} & \multirow{2}{*}{0,000} & \multirow{2}{*}{ sig. à 0,01} \\
\hline & Contrôle & 38 & 12,474 & 5,124 & & & \\
\hline \multirow{2}{*}{$\begin{array}{l}\text { Habiletés en } \\
\text { matière de } \\
\text { présentation }\end{array}$} & Expérimental & 38 & 11,816 & 2,577 & \multirow{2}{*}{4,024} & \multirow{2}{*}{0,000} & \multirow{2}{*}{ sig. à 0,01} \\
\hline & Contrôle & 38 & 8,737 & 3,950 & & & \\
\hline \multirow{2}{*}{$\begin{array}{l}\text { Gestion du } \\
\text { temps }\end{array}$} & Expérimental & 38 & 12,211 & 2,683 & \multirow{2}{*}{3,968} & \multirow{2}{*}{0,000} & \multirow{2}{*}{ sig. à 0,01} \\
\hline & Contrôle & 38 & 9,132 & 3,960 & & & \\
\hline \multirow{2}{*}{$\begin{array}{l}\text { Travail } \\
\text { d'équipe/en } \\
\text { groupe }\end{array}$} & Expérimental & 38 & 12,289 & 2,324 & \multirow{2}{*}{3,800} & \multirow{2}{*}{0,000} & \multirow{2}{*}{ sig. à 0,01} \\
\hline & Contrôle & 38 & 9,421 & 4,031 & & & \\
\hline \multirow{2}{*}{$\begin{array}{l}\text { Efficacité } \\
\text { personnelle }\end{array}$} & Expérimental & 38 & 14,842 & 3,054 & 3080 & 0000 & $\sin$ \\
\hline & Contrôle & 38 & 10,974 & 5,139 & דיק & 0,000 & II \\
\hline Esprit & Expérimental & 38 & 12,553 & 2,321 & 3,869 & 0,000 & sia à 001 \\
\hline d'initiative & Contrôle & 38 & 9,579 & 4,131 & & & \\
\hline Exprimer sa & Expérimental & 38 & 12,500 & 2,334 & 3951 & 0000 & sio à 001 \\
\hline alité & Contrôle & 38 & 9,395 & 4,246 & 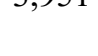 & 0,000 & Dig. a \\
\hline Deoré to & Expérimental & 38 & 147,895 & 24,309 & 4562 & 0,000 & 200 \\
\hline Degit total & Contrôle & 38 & 110,237 & 44,703 & 4,502 & 0,000 & Sig. a 0,01 \\
\hline
\end{tabular}

Valeur de la table $\mathrm{t}$ au dl (degré de liberté) 74 et au niveau de signification $0,05=2,00$ Valeur de la table $\mathrm{t}$ au dl (degré de liberté) 74 et au niveau de signification $0,01=2,66$ 
Comme le montre le tableau 3.1, la valeur obtenue pour $t(4,562)$ est supérieure à la valeur correspondante de la table $t(2,00)$, ce qui signifie qu'il existe des différences statistiquement significatives à $\alpha \leq 0,05$ dans la note moyenne totale figurant sur la grille d'observation post-expérimentation, entre le groupe expérimental et le groupe contrôle, le groupe expérimental affichant des notes plus élevées. La moyenne de la grille d'observation post-expérimentation dans le groupe expérimental atteignait 147,895, contre 110,237 dans le groupe contrôle. Ce résultat indique que l'utilisation de la stratégie d'apprentissage par projets est plus efficace que la méthode traditionnelle pour le développement des compétences en leadership des élèves.

La taille de l'effet de la stratégie d'apprentissage par projets sur le groupe expérimental, indiquée sur la grille d'observation post-expérimentation, a été mesurée et les résultats sont présentés dans le tableau 3.2.

Tableau 3.2 : Valeurs de $t, \eta 2$ et pour le degré total

\begin{tabular}{|c|c|c|c|c|}
\hline Compétences & $t$ & $\eta^{2}$ & $d$ & Taille de l'effet \\
\hline Planification & 4,339 & 0,203 & 1,009 & Grande \\
\hline Aptitude à communiquer & 4,388 & 0,206 & 1,020 & Grande \\
\hline $\begin{array}{l}\text { Compétences en résolution } \\
\text { de problèmes }\end{array}$ & 4,594 & 0,222 & 1,068 & Grande \\
\hline Prise de décisions & 3,922 & 0,172 & 0,912 & Grande \\
\hline Confiance en soi & 3,790 & 0,163 & 0,881 & Grande \\
\hline $\begin{array}{l}\text { Habiletés en matière de } \\
\text { présentation }\end{array}$ & 4,024 & 0,180 & 0,936 & Grande \\
\hline $\begin{array}{l}\text { Compétences en gestion du } \\
\text { temps }\end{array}$ & 3,968 & 0,175 & 0,922 & Grande \\
\hline Travail d'équipe/en groupe & 3,800 & 0,163 & 0,884 & Grande \\
\hline Efficacité personnelle & 3,989 & 0,177 & 0,928 & Grande \\
\hline Esprit d'initiative & 3,869 & 0,168 & 0,899 & Grande \\
\hline Prise de responsabilités & 3,951 & 0,174 & 0,919 & Grande \\
\hline Compétences en leadership & 4,562 & 0,220 & 1,061 & Grande \\
\hline
\end{tabular}


Les valeurs de $\eta^{2}$ et de $d$ sont indiquées dans le tableau 3.2 et soulignent la grande taille de l'effet de la stratégie d'apprentissage par projets dans le niveau total des compétences en leadership. Cela peut être attribué au plaisir procuré, à la motivation et aux circonstances d'apprentissage par projets créées dans la classe et résultant de la stratégie d'apprentissage par projets.

Réponse à la troisième question de recherche. La troisième question était la suivante : Quelles sont les différences statistiquement significatives $(\alpha \leq 0,05)$ dans le niveau des compétences en leadership des élèves du groupe expérimental entre les stades initial et final de l'expérimentation? Pour répondre à cette question, le chercheur a testé l'hypothèse nulle selon laquelle il n'y a aucune différence statistiquement significative à $\alpha \leq 0,05$ dans le niveau des compétences en leadership des élèves du groupe expérimental, entre la grille d'observation préapplication et la grille d'observation post-expérimentation. Pour examiner cette hypothèse, les notes moyennes des résultats du groupe expérimental figurant sur la grille d'observation préapplication et sur la grille d'observation postexpérimentation ont été calculées. Un test $t$ des échantillons appariés a été utilisé pour analyser les données. Les résultats sont présentés dans le tableau 3.3.

Tableau 3.3 : Résultats des tests $t$ des échantillons appariés - Différences entre la grille d'observation préapplication et la grille d'observation post-expérimentation du groupe expérimental

\begin{tabular}{lccccccc}
\hline Compétence & Groupe & $\mathrm{N}$ & Moyenne & Écart-type & $t$ & $\begin{array}{c}\text { Valeur de } \\
\text { signification }\end{array}$ & $\begin{array}{c}\text { Niveau de } \\
\text { signification }\end{array}$ \\
\hline \hline Planification & Prétest & 38 & 11,3158 & 3,662 & 9,573 & 0,000 & sig. à 0,01 \\
& Post-test & 38 & 16,2105 & 3,580 & & & \\
Aptitude à & Prétest & 38 & 11,5526 & 3,151 & 7,334 & 0,000 & sig. à 0,01 \\
communiquer & Post-test & 38 & 16,5000 & 3,415 & & & \\
$\begin{array}{l}\text { Compétences } \\
\text { en résolution }\end{array}$ & Prétest & 38 & 7,6053 & 2,955 & & & \\
de problèmes & Post-test & 38 & 11,2632 & 2,956 & & & sig. à 0,01 \\
$\begin{array}{l}\text { Prise de } \\
\text { décisions }\end{array}$ & Prétest & 38 & 7,2632 & 2,544 & & & \\
$\begin{array}{l}\text { Confiance en } \\
\text { soi }\end{array}$ & Prétest & 38 & 11,6842 & 3,103 & & & sig. à 0,01 \\
$\begin{array}{l}\text { Habiletés en } \\
\text { matière de } \\
\text { présentation }\end{array}$ & Post-test & 38 & 16,2895 & 3,502 & 12,904 & 0,000 & sig. à 0,01 \\
\hline
\end{tabular}




\begin{tabular}{|c|c|c|c|c|c|c|c|}
\hline Compétence & Groupe & $\mathrm{N}$ & Moyenne & Écart-type & $t$ & $\begin{array}{l}\text { Valeur de } \\
\text { signification }\end{array}$ & $\begin{array}{l}\text { Niveau de } \\
\text { signification }\end{array}$ \\
\hline \multirow{2}{*}{$\begin{array}{l}\text { Compétences } \\
\text { en gestion du } \\
\text { temps }\end{array}$} & Prétest & 38 & 8,5263 & 2,128 & \multirow[b]{2}{*}{8,499} & \multirow[b]{2}{*}{0,000} & \multirow[b]{2}{*}{ sig. à 0,01} \\
\hline & Post-test & 38 & 12,2105 & 2,683 & & & \\
\hline \multirow{2}{*}{$\begin{array}{l}\text { Travail } \\
\text { d'équipe/en } \\
\text { groupe }\end{array}$} & Prétest & 38 & 9,3947 & 2,636 & \multirow[b]{2}{*}{9,844} & \multirow[b]{2}{*}{0,000} & \multirow[b]{2}{*}{ sig. à 0,01} \\
\hline & Post-test & 38 & 12,2895 & 2,324 & & & \\
\hline \multirow{2}{*}{$\begin{array}{l}\text { Efficacité } \\
\text { personnelle }\end{array}$} & Prétest & 38 & 10,2895 & 2,710 & \multirow{2}{*}{10,479} & \multirow{2}{*}{0,000} & \multirow{2}{*}{ sig. à 0,01} \\
\hline & Post-test & 38 & 14,8421 & 3,054 & & & \\
\hline \multirow{2}{*}{$\begin{array}{l}\text { Esprit } \\
\text { d'initiative }\end{array}$} & Prétest & 38 & 8,5789 & 2,213 & \multirow{2}{*}{11,744} & \multirow{2}{*}{0,000} & \multirow{2}{*}{ sig. à 0,01} \\
\hline & Post-test & 38 & 12,5526 & 2,321 & & & \\
\hline \multirow{2}{*}{$\begin{array}{l}\text { Prise de } \\
\text { responsabilités }\end{array}$} & Prétest & 38 & 8,7368 & 2,901 & \multirow{2}{*}{7,327} & \multirow{2}{*}{0,000} & \multirow{2}{*}{ sig. à 0,01} \\
\hline & Post-test & 38 & 12,5000 & 2,334 & & & \\
\hline \multirow{2}{*}{$\begin{array}{l}\text { Compétences } \\
\text { en leadership }\end{array}$} & Prétest & 38 & 103,1053 & 23,625 & \multirow{2}{*}{17,164} & \multirow{2}{*}{0,000} & \multirow{2}{*}{ sig. à 0,01} \\
\hline & Post-test & 38 & 147,8947 & 24,309 & & & \\
\hline
\end{tabular}

Valeur de la table $t$ au dl (degré de liberté) 37 et au niveau de signification $0,05=2,02$ Valeur de la table $t$ au dl (degré de liberté) 37 et au niveau de signification $0,01=2,70$

Comme le montre le tableau 3.3, la valeur obtenue pour $t(17,164)$ est supérieure à la valeur correspondante dans la table $t(2,02)$, ce qui signifie qu'il existe des différences statistiquement significatives à $\alpha \leq 0,05$ entre les notes moyennes préapplication et les notes moyennes post-expérimentation dans les compétences en leadership du groupe expérimental, les notes post-expérimentation étant plus élevées. Cela montre que l'utilisation de la stratégie d'apprentissage par projets améliore les compétences en leadership parmi les élèves. L'hypothèse nulle est donc rejetée.

Pour mesurer la taille de l'effet de la stratégie d'apprentissage par projets sur le groupe expérimental sur la grille d'observation post-expérimentation, nous avons testé la taille de l'effet, comme le montre le tableau 3.4.

Tableau 3.4 : Valeurs de $t, \eta 2$ et pour le degré total

\begin{tabular}{lcccc}
\hline \multicolumn{1}{c}{ Compétence } & $t$ & $\eta^{2}$ & $d$ & Taille de l'effet \\
\hline \hline Planification & 9,573 & 0,712 & 3,148 & Grande \\
Aptitude à communiquer & 7,334 & 0,592 & 2,411 & Grande \\
$\begin{array}{l}\text { Compétences en résolution } \\
\text { de problèmes }\end{array}$ & 7,709 & 0,616 & 2,535 & Grande \\
\hline
\end{tabular}




\begin{tabular}{|c|c|c|c|c|}
\hline Compétence & $t$ & $\eta^{2}$ & $d$ & Taille de l'effet \\
\hline Prise de décisions & 8,251 & 0,648 & 2,713 & Grande \\
\hline Confiance en soi & 12,904 & 0,818 & 4,243 & Grande \\
\hline $\begin{array}{l}\text { Habiletés en matière de } \\
\text { présentation }\end{array}$ & 6,626 & 0,543 & 2,178 & Grande \\
\hline $\begin{array}{l}\text { Compétences en gestion du } \\
\text { temps }\end{array}$ & 8,499 & 0,661 & 2,794 & Grande \\
\hline Travail d'équipe/en groupe & 9,844 & 0,724 & 3,237 & Grande \\
\hline Efficacité personnelle & 10,479 & 0,748 & 3,445 & Grande \\
\hline Esprit d'initiative & 11,744 & 0,788 & 3,861 & Grande \\
\hline Prise de responsabilités & 7,327 & 0,592 & 2,409 & Grande \\
\hline Compétences en leadership & 17,164 & 0,888 & 5,643 & Grande \\
\hline
\end{tabular}

Les valeurs de $\eta^{2}$ et de $d$ indiquées dans le tableau 3.4 montrent la grande taille de l'effet de la stratégie d'apprentissage par projets dans le degré total des compétences en leadership.

Réponse à la quatrième question de recherche. La quatrième question était la suivante : Observe-t-on des différences statistiquement significatives $(\alpha \leq 0,05)$ dans le niveau des compétences en leadership entre les élèves de sexe masculin et les élèves de sexe féminin du groupe expérimental? Pour répondre à cette question, nous avons testé l'hypothèse selon laquelle il existe des différences statistiquement significatives $(\alpha \leq 0,05)$ entre les garçons et les filles dans le niveau des compétences en leadership des élèves du groupe expérimental. Pour examiner cette hypothèse, les moyennes et les écarts-types des résultats des élèves garçons et filles ont été calculés sur la grille d'observation post-expérimentation. Un test $t$ des échantillons indépendants a été utilisé pour évaluer les différences significatives. Les résultats sont présentés dans le tableau 3.5. 
Tableau 3.5: Résultats des tests $t$ des échantillons indépendants - Différences entre les élèves de sexe masculin et les élèves de sexe féminin du groupe expérimental

\begin{tabular}{|c|c|c|c|c|c|c|c|}
\hline Compétence & Sexe & $\mathrm{N}$ & Moyenne & Écart-type & $t$ & $\begin{array}{l}\text { Valeur de } \\
\text { signification }\end{array}$ & $\begin{array}{l}\text { Niveau de } \\
\text { signification }\end{array}$ \\
\hline \multirow{2}{*}{ Planification } & Masc. & 16 & 17,000 & 2,828 & \multirow{2}{*}{1,165} & \multirow{2}{*}{0,252} & \multirow{2}{*}{ Aucune sig. } \\
\hline & Fém. & 22 & 15,636 & 4,006 & & & \\
\hline \multirow{2}{*}{$\begin{array}{l}\text { Aptitude à } \\
\text { communiquer }\end{array}$} & Masc. & 16 & 17,125 & 2,754 & \multirow{2}{*}{0,961} & \multirow{2}{*}{0,343} & \multirow{2}{*}{ Aucune sig. } \\
\hline & Fém. & 22 & 16,045 & 3,823 & & & \\
\hline \multirow{2}{*}{$\begin{array}{l}\text { Compétences en } \\
\text { résolution de } \\
\text { problèmes }\end{array}$} & Masc. & 16 & 11,750 & 2,793 & \multirow[b]{2}{*}{0,863} & \multirow[b]{2}{*}{0,394} & \multirow[b]{2}{*}{ Aucune sig. } \\
\hline & Fém. & 22 & 10,909 & 3,085 & & & \\
\hline \multirow{2}{*}{$\begin{array}{l}\text { Prise de } \\
\text { décisions }\end{array}$} & Masc. & 16 & 12,250 & 2,017 & \multirow{2}{*}{1,795} & \multirow{2}{*}{0,081} & \multirow{2}{*}{ Aucune sig. } \\
\hline & Fém. & 22 & 10,818 & 2,684 & & & \\
\hline \multirow{2}{*}{$\begin{array}{l}\text { Confiance en } \\
\text { soi }\end{array}$} & Masc. & 16 & 16,938 & 2,792 & \multirow{2}{*}{0,972} & \multirow{2}{*}{0,338} & \multirow{2}{*}{ Aucune sig. } \\
\hline & Fém. & 22 & 15,818 & 3,936 & & & \\
\hline \multirow{2}{*}{$\begin{array}{l}\text { Habiletés en } \\
\text { matière de } \\
\text { présentation }\end{array}$} & Masc. & 16 & 11,938 & 2,516 & \multirow{2}{*}{0,245} & \multirow{2}{*}{0,808} & \multirow{2}{*}{ Aucune sig. } \\
\hline & Fém. & 22 & 11,727 & 2,676 & & & \\
\hline \multirow{2}{*}{$\begin{array}{l}\text { Compétences en } \\
\text { gestion du } \\
\text { temps }\end{array}$} & Masc. & 16 & 12,625 & 2,306 & \multirow{2}{*}{0,808} & \multirow{2}{*}{0,424} & \multirow{2}{*}{ Aucune sig. } \\
\hline & Fém. & 22 & 11,909 & 2,942 & & & \\
\hline \multirow{2}{*}{$\begin{array}{l}\text { Travail } \\
\text { d'équipe/en } \\
\text { groupe }\end{array}$} & Masc. & 16 & 12,125 & 2,156 & \multirow{2}{*}{-} & \multirow{2}{*}{0,715} & \multirow{2}{*}{ Aucune sig. } \\
\hline & Fém. & 22 & 12,409 & 2,482 & & & \\
\hline \multirow{2}{*}{$\begin{array}{l}\text { Efficacité } \\
\text { personnelle }\end{array}$} & Masc. & 16 & 15,063 & 2,265 & 0375 & 0710 & Aucune sio \\
\hline & Fém. & 22 & 14,682 & 3,564 & תונד, & $0, \pi$ & Aucume sig. \\
\hline Esprit & Masc. & 16 & 13,125 & 1,857 & 1300 & 0108 & A urune si \\
\hline d'initiative & Fém. & 22 & 12,136 & 2,569 & 1,009 & 0,199 & Aucune sig. \\
\hline Prise de & Masc. & 16 & 12,625 & 2,217 & (2078 & 0703 & $A_{1}$ \\
\hline responsabilités & Fém. & 22 & 12,409 & 2,462 & $0,2,10$ & $0, / 03$ & Aucume sig. \\
\hline Compétences en & Masc. & 16 & 152,563 & 20,202 & 1010 & 0319 & Aucune sio \\
\hline leadership & Fém. & 22 & 144,500 & 26,850 & 1,010 & הונד, & Ruture sig. \\
\hline
\end{tabular}

Valeur de la table $t$ au dl (degré de liberté) 36 et au niveau de signification $0,05=2,02$ Valeur de la table $t$ au dl (degré de liberté) 36 et au niveau de signification $0,01=2,70$

Comme le montre le tableau 3.5, la valeur obtenue pour $t(1,010)$ est inférieure à la valeur correspondante dans la table $t(2,02)$, ce qui signifie qu'il n'y a pas de différence statistiquement significative à $\alpha \leq 0,01$ qui soit liée au sexe dans le niveau de compétences 
en leadership parmi les élèves du groupe expérimental sur la grille d'observation postexpérimentation.

\section{Discussion}

Cette section traite des résultats de l'étude. Elle résume les conclusions qui sont tirées des résultats. Elle contient également des suggestions et des recommandations pour une étude plus approfondie, ainsi que d'autres recommandations qui pourraient s'avérer utiles aux concepteurs de cours, aux professeurs d'anglais, aux superviseurs, aux élèves et aux éducateurs.

\section{Constatations de l'étude}

$\mathrm{Au}$ vu des résultats de cette étude, des différences statistiquement significatives ont été relevées à $\alpha \leq 0,01$ dans la note moyenne totale attribuée aux compétences en leadership sur la grille d'observation post-expérimentation, entre le groupe expérimental et le groupe contrôle, au profit du groupe expérimental. De plus, des différences statistiquement significatives ont été relevées à $\alpha \leq 0,05$ entre les notes des compétences en leadership figurant sur les grilles d'observation préapplication et celles figurant sur la grille d'observation post-expérimentation, dans le groupe expérimental. Par ailleurs, aucune différence statistiquement significative liée au sexe n'a été relevée à $\alpha \leq 0,01$ dans le niveau des compétences en leadership des élèves du groupe expérimental sur les grilles d'observation post-expérimentation. En sommes, les résultats globaux de l'étude ont montré que le groupe expérimental, qui a vécu la stratégie d'apprentissage par projets, a obtenu de meilleurs résultats que le groupe contrôle, à qui nous avons enseigné avec les méthodes traditionnelles. En conclusion, après six semaines d'expérimentation, tous les élèves du groupe expérimental ont montré de nettes améliorations de leurs compétences en leadership après la mise en œuvre de la stratégie d'apprentissage par projets, comme le montre la grille d'observation post-expérimentation.

Discussion sur les constatations relatives à la première et à la deuxième hypothèse. Comme la première et la deuxième hypothèse sont étroitement liées, nous allons donc les traiter ensemble. Les résultats ont montré des différences statistiquement significatives à $\alpha$ $\leq$ 0,01 dans la note moyenne totale attribuée aux compétences en leadership sur la grille d'observation post-expérimentation, entre le groupe expérimental et le groupe contrôle, le groupe expérimental affichant des notes plus élevées. Les constatations ont également montré des différences statistiquement significatives à $\alpha \leq 0,05$ dans les notes moyennes entre la grille d'observation préapplication et la grille d'observation post-expérimentation du groupe expérimental, la grille d'observation post-expérimentation affichant des notes 
de leadership plus élevées. De plus, le chercheur a observé que la taille de l'effet était grande dans les notes totales de la grille d'observation post-expérimentation. L'efficacité de la stratégie d'apprentissage par projets pour le développement des compétences en leadership des élèves et la grande taille de l'effet pourraient être attribuées à six facteurs.

Premièrement, les activités et les tâches que les élèves devaient accomplir étaient simples et adaptées à leur âge et à leurs particularités (annexe 2). Ces activités et tâches comprenaient beaucoup de mouvement et de nombreuses interactions, ce qui a aidé les élèves à les exécuter et à les terminer facilement. Cela a stimulé la confiance en soi des élèves et les a encouragés à exprimer leurs opinions et leurs sentiments sans hésitation.

Deuxièmement, la mise en œuvre des projets a fourni aux élèves l'occasion de participer à l'étape de planification, au cours de laquelle ils devaient fixer les objectifs et préparer le matériel requis pour chaque projet aux stades initiaux de la mise en œuvre de chaque projet. Cette stratégie a aidé à améliorer leur compétence en planification, qui est l'une des plus importantes compétences en leadership.

Troisièmement, durant les projets, les élèves communiquaient entre eux de façon positive, poliment et amicalement, afin d'atteindre les principaux objectifs du projet. De plus, ils ont partagé et échangé des idées et de l'information en faisant appel à des aptitudes efficaces verbales, non verbales et en communication écrite. Ils participaient également davantage aux discussions sur leurs projets avec les membres du groupe. Leurs aptitudes à communiquer ont ainsi été développées grâce à la stratégie d'apprentissage par projets.

Quatrièmement, les élèves devaient accomplir chaque activité dans un certain délai, ce qui les a amenés à diviser chaque tâche ou activité en plus petites tâches afin de les terminer en temps voulu. L'enseignante leur apprenait à utiliser cette compétence et les encourageait à créer un environnement compétitif qui a amélioré leur compétence en gestion du temps, considérée comme une compétence en leadership importante.

Cinquièmement, tous les élèves, même ceux qui étaient moins doués, étaient motivés et enthousiasmés par les activités. Ils se précipitaient tous pour participer efficacement en apportant le matériel nécessaire, en s'aidant mutuellement, en assumant différents rôles dans la classe, et en n'hésitant pas à poser des questions à l'enseignante. Cela a stimulé leur esprit d'initiative, une compétence nécessaire pour être un leader efficace.

Sixièmement, chaque projet devait être présenté. Les membres du groupe devaient se préparer pour cette présentation et choisir le leader chargé de présenter le projet. Un leader différent était choisi pour chaque présentation de projet, ce qui a fourni l'occasion à de 
nombreux élèves de jouer le rôle de présentateur. L'élève qui présentait le projet savait qu'il devait utiliser le langage corporel efficacement, faire les gestes qui convenaient et s'adresser à son auditoire en toute confiance pour expliquer son projet. Ces habiletés en matière de présentation, qui sont essentielles pour être un leader efficace, ont été nettement développées par de nombreux élèves du groupe expérimental.

Finalement, les résultats ont révélé des changements dans les compétences en leadership des élèves. Les observations de chercheur étaient en accord avec celles de l'autre enseignante, ce qui a montré que les résultats étaient exacts. Ces résultats appuient l'efficacité de l'apprentissage par projets pour l'acquisition des compétences en leadership et sont en accord avec les constatations d'Al-Jamal (2014), qui a observé que l'apprentissage par projets améliorait la propension des élèves à créer une communauté d'apprenants autonomes, et avec les constatations de Bagheri, et coll. (2013), selon lesquelles l'apprentissage par projets stimulait l'aptitude à l'apprentissage autonome, qui est l'une des compétences en leadership.

Discussion sur les constatations relatives à la troisième hypothèse. Les constatations relatives à la troisième hypothèse ont montré qu'il n'y avait aucune différence statistiquement significative liée au sexe à $\alpha \leq 0,01$ dans le niveau des compétences en leadership des élèves du groupe expérimental sur la grille d'observation postexpérimentation. Ce résultat pourrait être attribué à deux facteurs.

D'abord, les activités ont été reparties de façon égale parmi tous les élèves, et chaque élève a dû jouer un rôle pour atteindre les objectifs du projet. L'enseignante et la chercheuse principale ne faisaient aucune différence entre les élèves de sexe masculin et les élèves de sexe féminin lorsqu'elles répartissaient les activités. De plus, les groupes d'élèves étaient mixtes. Ensuite, tous les élèves étaient du même âge.

\section{Conclusion}

Les élèves étaient nettement plus engagés avec la stratégie APP qu'avec la méthode traditionnelle, la stratégie APP semblait les intéresser davantage et captait leur attention plus longtemps. Nos résultats semblent aussi montrer que la stratégie APP augmentait la motivation des élèves et leur participation en classe. Aussi, les interactions en classe entre les élèves semblent être augmentées. Sur le plan du développement, nos résultats suggèrent que la stratégie APP aidait les élèves à développer leurs compétences intellectuel, social, émotionnel et moral, qui sont des habiletés fondamentales que les apprenants doivent développer à l'école. De plus, elle semble aider les élèves à développer de nombreuses compétences en matière de capacité de raisonnement, comme la pensée critique, la 
créativité et les compétences en recherche. À la lumière des constatations de cette étude, nous suggèrons de mener d'autres études liées à l'effet de l'apprentissage par projets sur le développement de différentes compétences en matière de capacité de raisonnement. Il serait aussi perinant de mener des études similaires sur différentes matières scolaires et d'explorer les effets de l'apprentissage par projets sur le développement de différentes compétences en leadership des enfants en école maternelle.

\section{Références}

Adair, J. E. (2010). Effective strategic leadership: The complete guide to strategic management. Pan Books.

Alshaya, H., \& Oyaid, A. (2015). The Use of GOOGLE+ in Project Based Learning with Students of Princess Nourah University and their Satisfaction Towards it. Special International Educational Journal, 4 (1), 527-543.

Ardeo, J. M. G. (2003). Attitude towards English and ESP acquisition as an L2 or L3 at university. Ibérica: Revista de La Asociación Europea de Lenguas Para Fines Especificos (AELFE), 6, 109-133.

Astin, H. S., \& Astin, A. W. (1996). A Social Change Model of Leadership Development (University of California).

Ayto, J. (1999). Twentieth century words: The story of the new words in English over the last hundred years. Oxford University Press.

Bagheri, M., Ali, W. Z. W., Abdullah, M. C. B., \& Daud, S. M. (2013). Effects of projectbased learning strategy on self-directed learning skills of educational technology students. Contemporary Educational Technology, 4(1), 15-29.

Baş, G. (2011). Investigating the effects of project-based learning on students' academic achievement and attitudes towards English lesson. The Online Journal Of New Horizons In Education, 1(4), 1-15.

Bisland, A. (2004). Developing leadership skills in young gifted students. Gifted Child Today, 27(1), 24-27.

Blumenfeld, P. C., Soloway, E., Marx, R. W., Krajcik, J. S., Guzdial, M., \& Palincsar, A. (1991). Motivating project-based learning: Sustaining the doing, supporting the learning. Educational Psychologist, 26(3-4), 369-398.

Boudersa, N., \& Hamada, H. (2015). Student-Centered Teaching Practices: Focus on The Project-Based Model to Teaching in the Algerian High-School Contexts. Arab World English Journal, 25-41.

Branch, L. J. (2015). The impact of project-based learning and technology on student achievement in mathematics. In W. W. K. Ma., A. H. K. Yuen, J. Park, W. W. F. Lau, \& L. Deng (eds), New media, knowledge practices and multiliteracies (pp. 259268). Springer. 
Cameron, L. (2001). Children learning a foreign language. Lynne Cameron, Teaching Languages to Young Learners, 1-20.

Chang, C.-S., Wong, W.-T., \& Chang, C.-Y. (2011). Integration of project-based learning strategy with mobile learning: Case study of mangrove wetland ecology exploration project. Journal of Applied Science and Engineering, 14(3), 265-273.

Chard, S. C. (2013). The Project Approach to Teaching and Learning. https://www.communityplaythings.com/resources/articles/2013/the-projectapproach-to-teaching-and-learning

Chen, C.-H., \& Yang, Y.-C. (2019). Revisiting the effects of project-based learning on students' academic achievement: A meta-analysis investigating moderators. Educational Research Review, 26, 71-81. https://doi.org/10.1016/j.edurev.2018.11.001

Creghan, C., \& Adair-Creghan, K. (2015). The positive impact of project-based learning on attendance of an economically disadvantaged student population: A multiyear study. Interdisciplinary Journal of Problem-Based Learning, 9(2), 7.

Curtis-Bey, L. (2009). Project-based learning: Inspiring middle school students to engage in deep and active learning. NYC Department of Education.

Doğan, Y., Batdi, V., \& Yildirim, B. (2012). Teachers' Views on the Practice of ProjectBased Learning Approach in Primary School Science Education. New perspectives in science education, 5.

Eskrootchi, R., \& Oskrochi, G. R. (2010). A study of the efficacy of project-based learning integrated with computer-based simulation-STELLA. Educational Technology \& Society, 13(1), 236-245.

Foss, P., Carney, N., McDonald, K., \& Rooks, M. (2008). Project-based learning activities for short-term intensive English programs. The Philippine ESL Journal, 1, 57-76.

Fragoulis, I., \& Tsiplakides, I. (2009). Project-Based Learning in the Teaching of English as A Foreign Language in Greek Primary Schools: From Theory to Practice. English Language Teaching, 2(3), 113-119.

Gill, R. (2011). Theory and practice of leadership. Sage.

Hallermann, S., Larmer, J., \& Mergendoller, J. R. (2011). PBL in the elementary grades: Step-by-step guidance, tools and tips for standards-focused K-5 projects. Buck Institute for Education.

Harun, Y. B. (2006). Project-Based Learning handbook "educating the millennial learner"'. Educational Technology Division Ministry of Education, Malaysia.

Hay, I., \& Dempster, N. (2004). Student leadership development through general classroom activities. Educating: Weaving Research into Practice, 141-150.

Helm, J. H., \& Katz, L. G. (2016). Young investigators: The project approach in the early years. Teachers College Press. 
Hooper, A., \& Potter, J. (2020). Business of leadership: Adding lasting value to your organization. Routledge.

Jokinen, T. (2005). Global leadership competencies: A review and discussion. Journal of European Industrial Training, 29(3), 199-216. https://doi.org/10.1108/03090590510591085

Kennedy, J. (2015). Leadership Skills: Developing the Skills You Need to Lead. https://nls.ldls.org.uk/welcome.html?ark:/81055/vdc_100063642750.0x000001

Keshta, A. S. (2001). Alternative approaches for teaching English literature to undergraduate students in Gaza Strip. Houstan Press.

Kokotsaki, D., Menzies, V., \& Wiggins, A. (2016). Project-based learning: A review of the literature. Improving Schools, 19(3), 267-277. https://doi.org/10.1177/1365480216659733

Koparan, T., \& Güven, B. (2014). The Effect of Project Based Learning on the Statistical Literacy Levels of Student 8th Grade. European Journal of Educational Research, 3(3), 145-157.

Megayanti, T., Busono, T., \& Maknun, J. (2020). Project-based learning efficacy in vocational education: Literature review. IOP Conference Series: Materials Science and Engineering, 830, 042075. https://doi.org/10.1088/1757-899X/830/4/042075

Melesse, T. (2015). Differentiated instruction: Perceptions, practices and challenges of primary school teachers. Science, Technology and Arts Research Journal, 4(3), 253264.

Mergendoller, J. R., \& Thomas, J. W. (2001). Managing project-based learning: Principles from the field. Buck Institute for Education.

Mincemoyer, C. (2012). I can be a leader! Leadership Fun for Children. Better Kid Care. https://extension.psu.edu/programs/betterkidcare/knowledge-areas/environmentcurriculum/activities/all-activities/i-can-be-a-leader-leadership-fun-for-children

Moody, G. (2000). Using How People Learn Model with Leadership Skills Development. Arizona State University.

Nassir, S. M. S. (2014). The Effectiveness of Project-based Learning Strategy on Ninth Graders' Achievement Level and their Attitude towards English in Governmental Schools-North Governorate. The Islamic University of Gaza.

Nikolaeva, S. (2012). Improving initial teacher education by using the project-based approach. Educational Research eJournal, 1(1), 51-60.

Northouse, P. G. (2021). Introduction to leadership: Concepts and practice (Fifth edition). SAGE.

Patton, A. (2012). Work that matters: The teacher's guide to project-based learning. Paul Hamlyn Foundation.

Pont, B., Nusche, D., Moorman, H., \& Hopkins, D. (2008). Improving school leadership. OECD. 
Proulx, J. (2004). L'apprentissage par projet. Presses de l'Université du Québec.

Richards, J. C., Richards, J. C., \& Renandya, W. A. (2002). Methodology in language teaching: An anthology of current practice. Cambridge university press.

Ryan, J. (2010). Five Leadership skills you and Your Organization Can't do without. Center for Creative Leadership.

Satralkar, M. (2006). Child leadership. Training and Research Institute. INDUS.

Schroder, K. Noble, S. Christie, M. Goodman, S. Junes, G. and Rogers, R. (2001). Linking Leadership Development to Instruction. A Leadership Development Curriculum for Virginia Public Schools. Commonwealth of Virginia Department of Education.

Shafaei, A., Poorverdi, M., \& Parvizi, B. (2007). Use of project-based learning in increasing students' vocabulary knowledge \& communicative ability. In The Second Biennial International Conference on Teaching and Learning of English in Asia: Exploring New Frontiers (TELiA2) (pp. 1-11), 14-16 June 2007, Holiday Villa Beach \& Spa Resort, Langkawi. Faculty of Communication and Modern Languages, Universiti Utara Malaysia, Sintok.

Western, S. (2019). Leadership: A critical text. Sage.

Wilkins, D. A. (1972). Linguistics in language teaching. E. Arnold.

Xiong, R. (2008). Leadership in project management. Georgia Institute of Technology.

Yalcin, S. A., Turgut, U., \& Büyükkasap, E. (2009). The effect of project-based learning on science undergraduates' learning of electricity, attitude towards physics and scientific process skills. International Online Journal of Educational Sciences, 1(1), $81-105$.

Yule, G. (2005). The Study of Language (3rd ed.). Cambridge University Press. 


\section{Annexes}

\section{Annexe 1 : Grille d'observation}

\begin{tabular}{|c|c|c|c|c|c|c|c|}
\hline \multirow[t]{2}{*}{ No. } & \multirow{2}{*}{ Skill } & \multirow[t]{2}{*}{ Item } & \multicolumn{5}{|c|}{ Degree } \\
\hline & & & 1 & 2 & 3 & 4 & 5 \\
\hline \multirow[t]{4}{*}{1} & \multirow[t]{4}{*}{ Planning } & $\begin{array}{r}\text { The student can set the main } \\
\text { goals of the project. }\end{array}$ & & & & & \\
\hline & & $\begin{array}{r}\text { She can determine and prepare } \\
\text { the materials. }\end{array}$ & & & & & \\
\hline & & $\begin{array}{cc}\text { She can use alternative materials } & .3 \\
\text { and procedures to achieve the } & \text { goal. }\end{array}$ & & & & & \\
\hline & & $\begin{array}{l}\text { She can rearrange the activities } \\
\text { according to their importance. }\end{array}$ & & & & & \\
\hline \multirow[t]{4}{*}{2} & \multirow[t]{4}{*}{ Communication skills } & $\begin{array}{r}\text { She interacts with her colleagues } \\
\text { positively. }\end{array}$ & & & & & \\
\hline & & $\begin{array}{r}\text { She exchanges her colleagues the } \\
\text { opinions and ideas. }\end{array}$ & & & & & \\
\hline & & She speaks with other politely. .7 & & & & & \\
\hline & & She acts the role of teacher well. .8 & & & & & \\
\hline \multirow[t]{3}{*}{3} & \multirow[t]{3}{*}{ Problem-solving } & $\begin{array}{r}\begin{array}{r}\text { She can determine the problem } \\
\text { clearly. }\end{array} \\
\text { cle }\end{array}$ & & & & & \\
\hline & & $\begin{array}{c}\text { She can collect data about the } .10 \\
\text { problem. }\end{array}$ & & & & & \\
\hline & & $\begin{array}{c}\text { She can put many solutions to } .11 \\
\text { the problem. }\end{array}$ & & & & & \\
\hline \multirow[t]{3}{*}{4} & \multirow[t]{3}{*}{ Decision making } & $\begin{array}{c}\text { She searches for logical solutions } .12 \\
\text { to face the problem. }\end{array}$ & & & & & \\
\hline & & She chooses the best solution. .13 & & & & & \\
\hline & & $\begin{array}{c}\text { She makes decisions in the right } .14 \\
\text { time. }\end{array}$ & & & & & \\
\hline \multirow[t]{3}{*}{5} & \multirow[t]{3}{*}{ Self-confidence } & $\begin{array}{l}\text { She says her opinion } .15 \\
\text { confidently. }\end{array}$ & & & & & \\
\hline & & $\begin{array}{c}\text { She expresses her feelings } .16 \\
\text { without hesitation. }\end{array}$ & & & & & \\
\hline & & $\begin{array}{c}\text { She accepts the constructive } .17 \\
\text { criticism. }\end{array}$ & & & & & \\
\hline
\end{tabular}




\begin{tabular}{|c|c|c|c|c|c|c|}
\hline \multirow[t]{3}{*}{ No. } & \multirow[t]{3}{*}{ Skill } & \multirow[t]{2}{*}{ Item } & \multicolumn{4}{|c|}{ Degree } \\
\hline & & & 1 & 2 & 3 & 4 \\
\hline & & $\begin{array}{c}\text { She can face the audience } .18 \\
\text { confidently. }\end{array}$ & & & & \\
\hline \multirow[t]{3}{*}{6} & \multirow[t]{3}{*}{ Presentation skill } & She has linguistics fluency. .19 & & & & \\
\hline & & $\begin{array}{c}\text { She can use the body language } .20 \\
\text { properly. }\end{array}$ & & & & \\
\hline & & $\begin{array}{c}\text { She can attract and motivate } .21 \\
\text { others. }\end{array}$ & & & & \\
\hline \multirow[t]{3}{*}{7} & \multirow[t]{3}{*}{ Time management } & $\begin{array}{l}\text { She divides the activities } .22 \\
\text { according to the time. }\end{array}$ & & & & \\
\hline & & $\begin{array}{c}\text { She completes the tasks in the } .23 \\
\text { definite time. }\end{array}$ & & & & \\
\hline & & $\begin{array}{l}\text { She writes the tasks and } .24 \\
\text { activities in her own notebook. }\end{array}$ & & & & \\
\hline \multirow[t]{3}{*}{8} & \multirow[t]{3}{*}{ Team work } & $\begin{array}{c}\text { She doesn't hesitate helping her } .25 \\
\text { colleagues. }\end{array}$ & & & & \\
\hline & & She tends to work in groups. .26 & & & & \\
\hline & & $\begin{array}{c}\text { She works with others by team } .27 \\
\text { spirit. }\end{array}$ & & & & \\
\hline \multirow[t]{4}{*}{9} & \multirow{4}{*}{$\begin{array}{c}\text { Personal effectiveness } \\
\text { skill }\end{array}$} & She can persuade others easily. .28 & & & & \\
\hline & & $\begin{array}{c}\text { She is interested of others' } .29 \\
\text { feelings. }\end{array}$ & & & & \\
\hline & & She always encourages others. .30 & & & & \\
\hline & & $\begin{array}{c}\text { Her colleagues usually emulate } .31 \\
\text { her. }\end{array}$ & & & & \\
\hline \multirow[t]{3}{*}{10} & \multirow[t]{3}{*}{ Initiation } & She tends to help others quickly. .32 & & & & \\
\hline & & $\begin{array}{c}\text { She acts different roles inside the } .33 \\
\text { class. }\end{array}$ & & & & \\
\hline & & $\begin{array}{c}\text { She tends to ask the teacher } .34 \\
\text { questions. }\end{array}$ & & & & \\
\hline \multirow[t]{3}{*}{11} & \multirow[t]{3}{*}{$\begin{array}{l}\text { Opportunities for } \\
\text { responsibilities }\end{array}$} & $\begin{array}{l}\text { She depends on herself to } .35 \\
\text { complete the mission. }\end{array}$ & & & & \\
\hline & & $\begin{array}{r}\text { She does her best to achieve the } .36 \\
\text { goal. }\end{array}$ & & & & \\
\hline & & $\begin{array}{l}\text { She is responsible of her } .37 \\
\text { behaviors and decisions. }\end{array}$ & & & & \\
\hline
\end{tabular}


Annexe 2 : Un exemple de leçon

Topic : At the Market

Project Title : Favourite Food Questionnaire

\section{Objectives:}

At the end of this project students are expected to:

- identify the vocabulary of food.

- use the food words to express their likes and dislikes.

- describe others' likes and dislikes.

\section{Vocabulary:}

apples - figs - onions - melons - oranges - carrots - tomatoes - bananas grapes - potatoes - food - chips - fish - biscuits - chicken - ice cream - meat - lemons

\section{Structures:}

I like ....... (; I don't like .......

\section{Strategies :}

- Group work

- Pair work

- Brain storming

\section{Time :}

\section{Six sessions among 2 weeks}

\section{Materials :}

- Pictures of food

- Papers

- Pens

- Posters
- Scissors

- Glue

- Flip chart

- Work sheets

\begin{tabular}{|c|c|c|}
\hline Step & \multicolumn{1}{|c|}{ Teacher Role } & student Role \\
\hline Procedures : & • $\begin{array}{l}\text { Students describe what they } \\
\text { can see in groups. }\end{array}$ \\
\hline $\begin{array}{c}\text { Define } \\
\text { Problem and } \\
\text { Task }\end{array}$ & Warming up:
\end{tabular}




\begin{tabular}{|c|c|c|}
\hline Step & Teacher Role & student Role \\
\hline & $\begin{array}{l}\text { - Teacher displays a picture of } \\
\text { several types of food at the } \\
\text { market. } \\
\text { Revision: } \\
\text { - Teacher distributes work sheet } \\
\text { No. (1). } \\
\text { - Teacher checks the answers. } \\
\text { Presentation: } \\
\text { - } \quad \text { Teacher presents the new } \\
\text { words using real objects, flash } \\
\text { cards and word cards. } \\
\text { carrots - onions - tomatoes - } \\
\text { - potatoes } \\
\text { Teacher draws } \odot \text { I like....... } \\
\text { Teacher discusses the main } \\
\text { topic of the project with } \\
\text { students. } \\
\text { Teacher discusses the goals of } \\
\text { the project. }\end{array}$ & $\begin{array}{l}\text { - Students say the words they } \\
\text { know in English and the } \\
\text { new words in Arabic. } \\
\text { - Students match each word } \\
\text { with its picture using work } \\
\text { sheet No. (1). } \\
\text { - Students play a matching } \\
\text { game } \\
\text { - Students work in pairs to } \\
\text { express themselves. } \\
\text { - Students share } \\
\text { discussion with the teacher. } \\
\text { Each group write the goals } \\
\text { of the project with the help } \\
\text { of the teacher. }\end{array}$ \\
\hline Develop a Plan & $\begin{array}{l}\text { Teacher distributes a simple } \\
\text { project proposal form that } \\
\text { includes the objectives, } \\
\text { production type, tie schedule } \\
\text { and the role of each member. } \\
\text { - Teacher divides the class in to } \\
7 \text { groups of } 5 \text { members. } \\
\text { - Teacher gives students their } \\
\text { tasks and appoints a leader for } \\
\text { each task. } \\
\text { Teacher discusses the project } \\
\text { plan with the group members. }\end{array}$ & $\begin{array}{l}\text { - Students work in groups to } \\
\text { write the plan with the help } \\
\text { of the teacher. } \\
\text { - Student 1: design the } \\
\text { questionnaire. } \\
\text { - Student 2\&3: go } \\
\text { outdoors to collect } \\
\text { information. } \\
\text { - Student 4: analyse the } \\
\text { information to describe } \\
\text { others. } \\
\text { - Student 5: present the } \\
\text { project. }\end{array}$ \\
\hline
\end{tabular}




\begin{tabular}{|c|c|c|}
\hline Step & Teacher Role & student Role \\
\hline $\begin{array}{l}\text { Investigate and } \\
\text { Implement }\end{array}$ & $\begin{array}{l}\text { - Teacher displays a poster as a } \\
\text { model of the questionnaire on } \\
\text { the board and starts to paste the } \\
\text { pictures and words of food. } \\
\text { - Teacher helps and monitors. } \\
\text { - Teacher discusses the next } \\
\text { step. }\end{array}$ & $\begin{array}{l}\text { - Each group has the } \\
\text { materials which they need } \\
\text { to design the questionnaire. } \\
\text { - Groups start to design their } \\
\text { own questionnaires by the } \\
\text { help of the leader. }\end{array}$ \\
\hline $\begin{array}{c}\text { Collect } \\
\text { Information }\end{array}$ & $\begin{array}{l}\text { - Teacher asks the leader of this } \\
\text { task to implement the } \\
\text { questionnaire on a member of } \\
\text { his group } \\
\text { - Teacher encourages students. }\end{array}$ & $\begin{array}{l}\text { - Student 1: What food do } \\
\text { you like? } \\
\text { - Student2: answers. } \\
\text { - Student1 records the } \\
\text { answers ticking in front the } \\
\text { favourite food. } \\
\text { - The leader of the second } \\
\text { task implements the } \\
\text { questionnaire on other } \\
\text { persons. For example, his } \\
\text { mum, dad, teacher or } \\
\text { headmaster. }\end{array}$ \\
\hline $\begin{array}{c}\text { Provide } \\
\text { Feedback }\end{array}$ & $\begin{array}{l}\text { - Teacher asks student4 from } \\
\text { each group to analyse the } \\
\text { information. } \\
\text { - Teacher distributes empty } \\
\text { posters. } \\
\text { - Teacher gives sentences as } \\
\text { examples. } \\
\text { - Teacher goes around checking } \\
\text { and helping. }\end{array}$ & $\begin{array}{l}\text { - Students come back to the } \\
\text { classroom with data they } \\
\text { have collected } \\
\text { - Students summarize all the } \\
\text { information related the } \\
\text { previous task with the } \\
\text { teacher. } \\
\text { - Students write sentences } \\
\text { describing the person they } \\
\text { have asked }\end{array}$ \\
\hline $\begin{array}{l}\text { Present the } \\
\text { Project }\end{array}$ & $\begin{array}{l}\text { - Teacher monitors and gives } \\
\text { help when necessary. } \\
\text { - Teacher encourages students. }\end{array}$ & $\begin{array}{l}\text { - Each group prepares its } \\
\text { poster. } \\
\text { - Student5 from each group } \\
\text { goes out to present the } \\
\text { project. } \\
\text { - The leader of this task } \\
\text { describes the likes and } \\
\text { dislikes using the }\end{array}$ \\
\hline
\end{tabular}




\begin{tabular}{|c|c|c|}
\hline Step & Teacher Role & \multicolumn{1}{c|}{ student Role } \\
\hline & & $\begin{array}{l}\text { questionnaire they ticked } \\
\text { and the poster they wrote. }\end{array}$ \\
\hline $\begin{array}{c}\text { Reflect up and } \\
\text { Evaluation }\end{array}$ & $\bullet \quad \begin{array}{c}\text { Teacher gives a work sheet. } \\
\text { Teacher checks the answers. }\end{array}$ & $\begin{array}{l}\text { Students discuss the } \\
\text { findings. } \\
\text { Students do the work sheet } \\
\text { as a summative evaluation. }\end{array}$ \\
\hline
\end{tabular}

